\title{
Impact of COVID-19 on Clinical Operations and Management of Patients in a Singapore Immunodermatology Unit during the 'Circuit-Breaker' Period and Beyond
}

\section{Dear Editor,}

COVID-19 has swept the world by storm, with Singapore implementing strict safe distancing measures from 7 April to 2 June 2020, termed 'circuit-breaker', to curb local spread of COVID-19. As of 14 December 2020, there are a total of 58,325 confirmed cases (total prevalence rate $1.02 \%$ ) and 29 deaths. $^{1}$

The National Skin Centre is a tertiary dermatological centre in Singapore, with the Immunodermatology unit seeing patients with immunologically mediated conditions, including but not limited to connective tissue disorders, autoimmune blistering diseases (AIBD) and vasculitis. We discuss the impact of COVID-19 on our unit's clinical operations during the circuit-breaker period, and management of this group of patients, many who are on systemic immunosuppressive medications, during a pandemic.

Aquarter of our centre's staff — dermatologists, residents, nursing and allied health, were deployed to acute hospitals, holding facilities, and outbreak-affected migrant worker dormitories to augment manpower at the frontline of the COVID-19 pandemic. A team segregation model was implemented as part of centre-wide operational continuity plans. Should any staff be infected, or require a quarantine period, the unit's services suffer minimal disruption.

To ensure physical distancing, there was an urgent need to reduce patient load. To this end, patients who satisfy a set of criteria (Table 1) were proactively contacted, and offered teleconsultation via telephone/ video calls, or deferment of non-urgent clinic visits, with delivery of medications. We converted 4 (1.7\%) followup physical visits to teleconsultation in the circuit-breaker period. These patients did not require blood tests or vital signs monitoring. The low initial take-up rate were due to patients not fulfilling teleconsultation criteria, many elderly patients were not tech-savvy and could not obtain help from their family members if living in different households, and physicians' initial unfamiliarity with utilising this new mode of consultation. New patients who require skin biopsies as part of the diagnostic process are also not suitable for teleconsultation.
Table 1. Initial criteria for teleconsultations or postponement of appointments

\begin{tabular}{ll}
\hline Criteria \\
\hline $1 \quad \begin{array}{l}\text { Patients with mobility issues (such as being bed/trolley or } \\
\text { wheelchair-bound) or }\end{array}$ \\
\hline $2 \quad$ Age 65 and above \\
\hline And Condition is Stable With: \\
\hline \begin{tabular}{l} 
Patients on topical treatment only or \\
\hline 4
\end{tabular}$\quad \begin{array}{l}\text { Patients on monotherapy of low dose prednisolone } 15 \mathrm{mg} / \text { day or } \\
\text { lower } \text { or }\end{array}$ \\
\hline 5 & $\begin{array}{l}\text { Patients on low dose prednisolone } 15 \mathrm{mg} / \text { day or lower with } \\
\text { doxycycline }+/- \text { nicotinamide }\end{array}$ \\
\hline And & \\
\hline 6 & $\begin{array}{l}\text { Patients are not on other high alert medications e.g. } \\
\text { azathioprine, dapsone and mycophenolate mofetil }\end{array}$ \\
\hline
\end{tabular}

With these measures, the Immunodermatology unit's attendance during the circuit-breaker period fell by $33.4 \%$, compared to a similar period in 2019 . Average weekly default rates of pre-fixed appointments also doubled from $9.4 \%$ to $19.7 \%$, likely contributed by patients' reluctance to visit the clinic during the initial implementation of strict safe distancing measures. For patients who defaulted clinic visits and contacted the centre, short-term re-supply of medications were arranged and appointments rescheduled. For patients who continued to attend physical visits, courier service for medications was made available such that they could avoid waiting at a crowded pharmacy.

The need to balance risks of acquiring COVID-19 from immunosuppression has to be weighed with that of a flare of the dermatological condition, especially so in AIBD. We have seen several patients whose AIBD flared when they abruptly stopped medications after they defaulted visits due to fears of exposure to COVID-19. Some even had severe flares that required hospitalisation during the circuit-breaker period which, contrary to their intentions, may expose them unnecessarily to infection risks, also further straining the healthcare system. 
Conventional immunosuppressive agents commonly used in our patients include prednisolone, azathioprine, methotrexate and mycophenolate mofetil. While there is insufficient evidence to confirm that COVID-19 infection is worsened by immunosuppressive medications, such drugs result in broad suppression across the cytokine milieu, which may increase susceptibility to viral infections. ${ }^{2}$ In turn, infections may trigger flares of underlying $\mathrm{AIBD}^{3}$ or vasculitis. While we have yet to encounter confirmed COVID-19 cases among our patients, we continue to remain vigilant. We adopted the advice in international publications $^{2-5}$ to stop immunosuppressive agents when viral symptoms are present in patients with exposure to COVID-19, except for gradual tapering of corticosteroids to reduce risk of adrenal insufficiency. It is unclear when it is safe to resume these medications in patients with recent COVID-19 infection. One Italian dermatological clinic empirically advised patients to restart the drug when a negative COVID-19 swab result is obtained. ${ }^{3}$

In well patients without COVID-19 symptoms, we consider the severity of the underlying cutaneous diseases and other comorbidities when initiating and continuing immunosuppressive medications, and select less immunosuppressive oral medications or topical treatment wherever possible. ${ }^{4,5}$

There are no clear guidelines for patients on intravenous immunoglobulin, but as it has been proposed as an adjuvant treatment in COVID-19 infections, demonstrating mortality benefit, ${ }^{6,7}$ there may be no need to cease this therapy even when infected with COVID-19.

Our physicians' decisions to initiate, continue or up-titrate systemic immunosuppressive medications requiring laboratory monitoring have also been affected by logistical challenges amplified during this pandemic. It is a tussle between the potential myelo-, hepato-, or renal toxicity, and higher risk of cutaneous flare if therapy is withheld or suboptimal. As primary care clinics took on the responsibility of reviewing patients with acute respiratory symptoms and screening for COVID-19, directing our patients to these clinics for blood-taking during the circuit-breaker period was not ideal. Hence, face-to-face phlebotomy and consultations, even at close 2-3 weekly intervals, may be inevitably necessary.

Rituximab, an anti-CD20 antibody that is US Food and Drug Administration-approved for moderate-severe pemphigus, causes irreversible and prolonged depletion of B-cells, which may have serious effects, such as inducing severe COVID-19 pneumonia. ${ }^{8}$ As patients with AIBD who have been treated with rituximab within the last year may experience a worse or longer disease course of COVID-19 compared to healthy individuals, ${ }^{3}$ we are heeding the recommendations that rituximab infusions for patients with pemphigus be postponed to delay the peak of immunosuppression during the ongoing pandemic, ${ }^{9}$ instead opting for conventional agents with shorter half-lives (e.g. prednisolone, dapsone and azathioprine) in the interim. Rituximab, administered intravenously in hospitals, are also discouraged as beds are limited during a pandemic.

Many international dermatological centres affected by the outbreak have adjusted clinical workflows: instituting infection control measures, ${ }^{10,11}$ drastic reduction in clinics ${ }^{11}$ team segregation to limit cross-contamination between healthcare workers, ${ }^{12}$ triaging conditions that should be reviewed physically, ${ }^{13}$ and even temporarily ceasing operations. ${ }^{14}$

With resumption of healthcare services after the circuit-breaker period, the number of patients in the Immunodermatology unit is returning to pre-pandemic load. To restrict crowding, the teleconsultation criteria have been revised. Age has been removed, and patients on high alert medications are allowed to replace some face-to-face visits with teleconsultation if blood tests are done in advance. Patients may consider going to primary care clinics for blood tests or engaging home phlebotomy services, and self-measurement of blood pressure and glucose. As household visitation restrictions have been lifted, family members who are tech-savvy can now assist patients for teleconsultations. The centre is also expanding doctor-to-doctor teleconsultation, and referral services in the primary care clinics and home care settings.

More than $70 \%$ of physicians surveyed in a study found it 'difficult' to diagnose conditions over teleconsultation compared to physical consultation, and that teleconsultations were more time-consuming for the doctors though more convenient for patients..$^{15}$ Of note, 'inflammatory dermatitis' ranked lowest in terms of ease of diagnosis over teleconsultations. ${ }^{15}$ To address physicians' concerns and difficulties in teleconsultation, ongoing centre-wide surveys are also being conducted in NSC to identify problems and improve workflow.

Beyond the circuit-breaker period, we are continually fine-tuning the triage and logistics of teleconsultations, to minimise crowding and ensure sufficient capacity for concurrent pandemic work, while providing up-to-date care. This pandemic has not only pressed us to adapt but also created opportunities to improve the delivery of care for our patients. 


\section{REFERENCES}

1. Ministry of Health, Singapore. Situation Report. Available at: https://www.moh.gov.sg/covid-19/situation-report. Accessed on 15 December 2020

2. Galimberti F, McBride J, Cronin M, et al. Evidenced-based best practice advice for patients treated with systemic immunosuppressants in relation to COVID-19. Clin Dermatol 2020 May 14.

3. Kasperkiewicz M, Schmidt E, Fairley J, et al. Expert recommendations for the management of autoimmune bullous diseases during the COVID-19 pandemic. J Eur Acad Dermatol Venereol 2020;34:e302-3.

4. Wang C, Rademaker M, Baker C, et al. COVID-19 and the use of immunomodulatory and biologic agents for severe cutaneous disease: an Australia/New Zealand consensus statement. Australas J Dermatol 2020;61:210-6.

5. National Institute for Health and Care Excellence. COVID-19 rapid guideline: dermatological conditions treated with drugs affecting the immune response. Available at: https://www.nice.org.uk/guidance/ ng169. Accessed on 27 May 2020.

6. Cao W, Liu X, Bai T, et al. High-dose intravenous immunoglobulin as a therapeutic option for deteriorating patients with coronavirus disease 2019. Open Forum Infect Dis 2020;7:ofaa102.

7. Xie $\mathrm{Y}$, Cao S, Dong $\mathrm{H}$, et al. Effect of regular intravenous immunoglobulin therapy on prognosis of severe pneumonia in patients with COVID-19. J Infect 2020;81:318-56.

8. Guilpain P, Le Bihan C, Foulongne V, et al. Rituximab for granulomatosis with polyangiitis in the pandemic of covid-19: lessons from a case with severe pneumonia. Ann Rheum Dis 2020 Apr 20;annrheumdis-2020-217549.
9. Shakshouk H, Daneshpazhooh M, Murrell DF, et al. Treatment considerations for patients with pemphigus during the COVID-19 pandemic. J Am Acad Dermatol 2020;82:e235-6.

10. Zheng Y, Lai W. Dermatology staff participate in fight against Covid-19 in China. J Eur Acad Dermatol Venereol 2020;34:e210-1.

11. Chen Y, Pradhan S, Xue S. What are we doing in the dermatology outpatient department amidst the raging of the 2019 novel coronavirus? J Am Acad Dermatol 2020;82:1034.

12. Price KN, Thiede R, Shi VY, et al. Strategic dermatology clinical operations during the coronavirus disease 2019 (COVID-19) pandemic. J Am Acad Dermatol 2020;82:e207-9.

13. Giacalone S, Bortoluzzi P, Nazzaro G. Which are the "emergent" dermatologic practices during COVID-19 pandemic? Report from the lockdown in Milan, Italy. Int J Dermatol 2020;59:e269-70.

14. Lim TK. The facts, fallacies and uncertainties about coronavirus disease 2019 (COVID-19). Ann Acad Med Singap 2020;49:343-5.

15. Sharma A, Jindal V, Singla $P$, et al. Will teledermatology be the silver lining during and after COVID-19? Dermatol Ther 2020;e13643.

Ziying V $\underline{\operatorname{Lim}},{ }^{1} M B B S, M R C P(U K), \operatorname{MMed}($ Int Med),

Yen Loo $\underline{\operatorname{Lim}},{ }^{1} M B B S, M R C P(U K), F R C P(E d i n)$

${ }^{1}$ Immunodermatology unit, National Skin Centre, Singapore

Address for Correspondence: Dr Vanessa Lim Ziying, National Skin Centre, 1 Mandalay Road, Singapore 308205.

Email: vlim@nsc.com.sg 